\title{
Coupled Mode Characteristics From the Perturbation of 3D Printed Long-Period Fiber Grating Devices
}

\author{
Ravivudh KHUN-IN ${ }^{*}$, Yuji USUDA ${ }^{1}$, Yuttapong JIRARAKSOPAKUN ${ }^{2}$, \\ Apichai BHATRANAND ${ }^{2}$, and Hideki YOKOI ${ }^{1}$
}

\author{
${ }^{1}$ Graduate School of Engineering and Science, Shibaura Institute of Technology, Tokyo 135-8548, Japan \\ ${ }^{2}$ Department of Electronics and Telecommunication Engineering, King Mongkut's University of Technology Thonburi, \\ Bangkok 10140, Thailand \\ *Corresponding author: Ravivudh KHUN-IN_Ｅ-mail: nb17509@shibaura-it.ac.jp
}

\begin{abstract}
Characteristics of electric field from a coupled mode inside an optical fiber under perturbation by three-dimensional (3D) printed long-period fiber grating (LPFG) device have been observed in this work by the experiment and simulation. The various periodic index differences referring to the weights of perturbation by 3D printed LPFG device are applied on the single-mode fiber. The experimental results show that the resonant wavelength shift is a linear function of the grating period with the maximum coefficient of determination $R^{2}$ of 0.9995 . Some of resonant wavelengths are chosen to run simulations to investigate the electric field distribution. The scattering direction of the electric field states the magnitude of leaking optical power when the light transmits through the grating region applied to the single-mode fiber. Both the experimental and simulation results demonstrate that our proposed scheme can usefully be applied to selective tunable filters, intruder sensors, etc.
\end{abstract}

Keywords: 3D printed long-period fiber grating device; perturbation; resonant wavelength; electric field distribution

Citation: Ravivudh KHUN-IN, Yuji USUDA, Yuttapong JIRARAKSOPAKUN, Apichai BHATRANAND, and Hideki YOKOI, "Coupled Mode Characteristics From the Perturbation of 3D Printed Long-Period Fiber Grating Devices," Photonic Sensors, 2020, 10(3): 195-203.

\section{Introduction}

Long-period fiber grating (LPFG) is one type of grating structures, in which its grating period is generated or induced inside an optical fiber. The periodic refractive index change inside fiber's core layer has a major roll to couple modes of light from a fundamental core mode to cladding modes [1]. The characteristics of these coupling actions are observed as resonant wavelengths or wavelength dips after the light is transmitted through the grating structure inside the optical fiber. Several techniques of LPFG fabrication have been researched to find their performances of coupling modes, in terms of resonant wavelengths such as phase mask technique [2] and $\mathrm{CO}_{2}$ laser technique [3]. Those aforementioned techniques permanently change the fiber structure when the structure of LPFG is formed by laser irradiation direct to the optical fiber. Besides there is another technique that temporarily changes the fiber structure, so that the optical fiber becomes LPFG only when the LPFG structure is

Received: 25 September 2019 / Revised: 15 January 2020

(C) The Author(s) 2020. This article is published with open access at Springerlink.com

DOI: $10.1007 / \mathrm{s} 13320-020-0585-2$

Article type: Regular 
applied on to the fiber, and that fiber can return to its initial state right after the LPFG structure is removed. Such a technique is called mechanically induced technique [4]. This technique uses an external device to create an LPFG structure inside the fiber core by pressing the LPFG on to the optical fiber.

The single-mode fiber is employed in this experiment because it yields very low attenuation and it is commonly used in telecommunication network. Only fundamental core mode is transmitting for the simple observation of the mode coupling. The 3D printed LPFG devices [5] designed with a variety of grating periods are used to observe the trend of the resonant wavelength shift from the perturbation. The conventional perturbation by using coiled spring [6] reported that the relationship between the grating period and the resonant wavelength was linear, but the coupled mode has not been observed by a simulation. The resonant wavelength shifts to longer wavelength with the longer applied grating period of LPFG device. In addition, the magnitude of resonant wavelength or its dip is also investigated from the weight of perturbation actioned by $3 \mathrm{D}$ printed LPFG device.

Aside from the experiment, the simulation throughout the grating period structure along the fiber axis is taken to observe the electric field distribution at the resonant wavelength by FullWAVE (Synopsys Inc.) software. The LPFG structure is designed in the initial single-mode fiber by stating the periodic refractive index differences from its initial refractive index of the core layer of the standard single-mode fiber. These periodic refractive index differences relate to the weight of the perturbation by the $3 \mathrm{D}$ printed LPFG device in the experiment. The electric field distribution along fiber's core layer is considered to the mode coupling actions referring to the experiment.

This paper presents the coupled mode characteristics from the perturbation of 3D printed LPFG devices using mechanically induced technique. The $3 \mathrm{D}$ printed LPFG device is used to create the grating perturbation on to the single-mode optical fiber and then the resonant wavelengths are observed with the help of a broadband light source emitting its maximum power locating at around $1550 \mathrm{~nm}$. In addition, the characteristics of electric field distribution from corresponding resonant wavelengths are investigated.

\section{3D printed LPFG device and the experimental setup}

\subsection{Principle of 3D printed LPFG device}

The popularity of a 3D printing technology has become increasing due to its resolution and materials. The printed material has been developed to possess high durability and remains its original shape under harsh environment or under strong perturbation. The material used in this work is a rigid translucent resin (Polyjet RGD720) and a printer is a high resolution 3D printer (Objet Connex500) with the initial resolution of $16 \mu \mathrm{m}$.

The structure of the 3D printed LPFG device is a rectangular shape with the dimension of $(25 \times 40 \times 5) \mathrm{mm}^{3}$. One of its surfaces with the dimension of $(40 \times 25) \mathrm{mm}^{2}$ is designed as triangular vertexes to generate a grating structure. Each vertex represents the grating pitch and the distance between each pitch represents a grating period. The length of the device is varied around $40 \mathrm{~mm}$ depending on the grating period. The structure of the device is initially designed using Solidworks software as shown in Fig. 1(a). This dimension of the 3D printed LPFG structure is specially designed to be compact in order to support the flat tip rod of the digital force meter during the perturbation process. The device with lower thickness easily bends when high force is directly applied on to the device. Even the vertexes surface designed in Solidworks is triangular shape, the printed device does not have perfectly triangular shape of vertexes as shown in a scanned electron microscope (SEM) image in Fig. 1(b). In addition, the precise length of the device corresponding to the grating period and the number of grating pitches are 
stated in Table 1. The number of grating pitches decreases while the grating period is longer to remain the length of device not longer than $40 \mathrm{~mm}$.

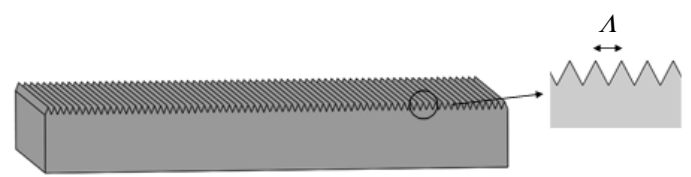

(a)

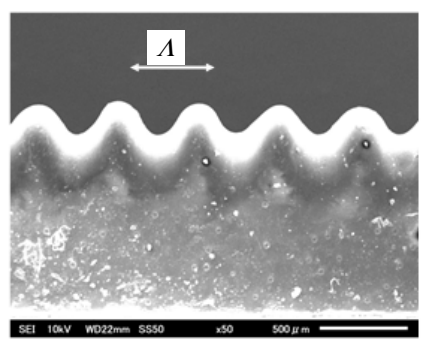

(b)

Fig. 1 3D printed LPFG device: (a) designed in Solidworks and (b) observed by SEM.

Table 1 Specification of 3D printed LPFG device with different grating periods.

\begin{tabular}{ccc}
\hline Grating period $(\mu \mathrm{m})$ & Grating length $(\mathrm{mm})$ & No. of pitches \\
\hline 520 & 39.52 & 76 \\
530 & 39.75 & 75 \\
540 & 39.96 & 74 \\
550 & 39.60 & 72 \\
560 & 39.76 & 71 \\
570 & 39.90 & 70 \\
580 & 39.44 & 68 \\
590 & 39.53 & 67 \\
600 & 39.60 & 66 \\
610 & 39.65 & 65 \\
\hline
\end{tabular}

\subsection{Mode coupling in LPFG}

Partial wavelength of light is attenuated, so called resonant wavelength, when it transmits through the periodic index difference inside core layer. The relationship between the resonant wavelength and grating period is expressed in terms of phase matching curve (PMC) as a linear equation by [7]

$$
\lambda_{\text {res }}=\left[n_{\text {eff,co }}-n_{\text {eff,cl }}^{i}\right] \Lambda
$$

where $\lambda_{\text {res }}$ and $\Lambda$ are the resonant wavelength and grating period, respectively. $n_{\text {eff,co }}$ is the effective index of core layer and $n_{\mathrm{eff}, \mathrm{cl}}^{i}$ is the effective index of cladding layer at Mode $i$. The complete coupling takes place when the mode field is in phase with the grating pitch inside an LPFG. The corresponding light still passes through grating pitch where the mode field is not in phase. The transmission of the attenuation band of the resonant wavelength is expressed by [8]

$$
T_{i}=1-\sin ^{2}\left(\kappa_{i} L\right)
$$

where $\kappa_{i}$ is the coupling coefficient at the $i$ th mode of cladding layer and $L$ represents the grating length. The light couples to cladding mode when it reaches the grating pitch that locates at phase position. When all transmitted lights are totally coupled to cladding mode, there is no any effect of the attenuation for longer grating length.

\subsection{Experimental setup}

The formation of LPFG on the single-mode fiber is illustrated in Fig. 2. The light transmitter is the super luminescent diode emitting light wave at $1550 \mathrm{~nm}$. The receiver is an optical spectrum analyzer (OSA) that can display the light spectrum in the range between $1250 \mathrm{~nm}$ and $1750 \mathrm{~nm}$ in the unit of $\mathrm{dBm}$. The bare single-mode fiber is used as a medium for light transmission and its partial region is altered to the LPFG structure. The fiber is put on the relaxation state as there is no strength or strain applied on the fiber and the temperature for the experiment is fixed at $25^{\circ} \mathrm{C}$. The $3 \mathrm{D}$ printed LPFG device is directly positioned on the bare fiber in which its length axis is parallel to the fiber axis. The perturbation process is taken by the digital force meter installed with the force stand. The LPFG structure is then formed on the bare fiber to generate the perturbation. To avoid the damage on the fiber, the fiber coating layer is not removed. The weights of perturbation read out by the digital force meter are applied to the LPFG device between $0.0 \mathrm{~kg}$ and $4.0 \mathrm{~kg}$ with the increment of $0.5 \mathrm{~kg}$. 


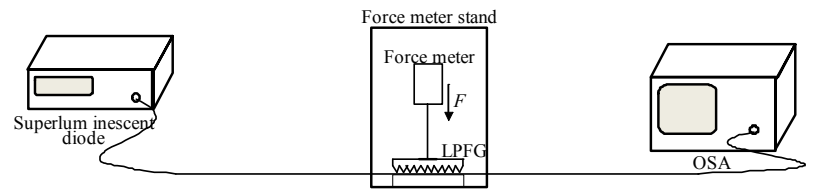

Fig. 2 Experimental setup of LPFG fabricated by 3D printed LPFG device.

\section{Electric field distribution along the optical fiber}

The ideal structure of LPFG illustrated in Fig. 3 is designed based on the experiment of grating perturbation. The electric field along the fiber core axis is observed by the simulation under finite-difference time-domain (FDTD) technique [9] of FullWAVE (Synopsys Inc.). The cylindrical structure refers to fiber's core layer. The refractive indices of the structure follow the specification of standard SMF-28 step-index fiber in which their refractive indices of core and cladding layer of the structure are set at 1.45205 and 1.44681, respectively [10]. The LPFG structure inside the core layer is designed up to only 3 grating pitches as the rest of remaining pitches from Table 1 give the same characteristics of the electric field. The red region refers to the initial refractive index of core layer without any applied force and the blue region refers to the region where the perturbation occurs. The purple boundary is the spatial domain for the region of simulation and the width is set to the default value of $10.62 \mu \mathrm{m}$ to every structure. Since the simulation of the large structure takes too much time to obtain the electric field distribution; therefore, many parameters have to be adjusted. The grating length has to be shortened in the simulation to only a few micrometers instead of $40 \mathrm{~mm}$ as used in the experiment. For instance, the grating period for the LPFG device of $5.3 \mu \mathrm{m}$ is used in the simulation instead of $530 \mu \mathrm{m}$ in the experiment. The fiber core diameter is also decreased to $0.8 \mu \mathrm{m}$ as the normal fiber core diameter is $8 \mu \mathrm{m}$. When the structure of an LPFG is shrunk to be much smaller than the real fiber, the refractive index of perturbation region or at the grating pitch has to be set up to a very high value at 1.84681 , according to the difference from its initial core index of 0.4. The environment surrounding that cylindrical structure represents the fiber's cladding layer. The fundamental core mode is coupled to cladding layer as seen in the form of electric field distribution when the light transmits through periodic index differences along the fiber core structure.

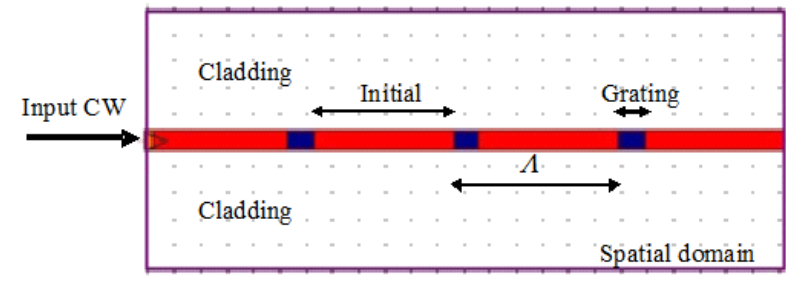

Fig. 3 LPFG structure in FullWAVE (Synopsys Inc.).

At the launching section, the continuous wave (CW) is used with the corresponding resonant wavelengths and the same grating periods from the experiment. For instance, the resonant wavelength appears at $1467 \mathrm{~nm}$ when the fiber is perturbed by using $520 \mu \mathrm{m}$ grating period device. Then, for the simulation, $1467 \mathrm{~nm}$ is used as an input for the LPFG structure with the length of area with a different refractive index (the length of red and blue areas in Fig. 3) of $5.2 \mu \mathrm{m}$. The structure design of the LPFG structure in FullWAVE (Synopsys Inc.) illustrated by a 2D planar in Fig. 3 is an example of the structure with a periodic index different distance within core layer of $5.2 \mu \mathrm{m}$. The length of each grating pitch is $1 \mu \mathrm{m}$. Therefore, the total length of the simulated structure is $19.8 \mu \mathrm{m}$. Input parameters to simulate the electric field distribution corresponding to the perturbation of LPFG device with grating periods of $520 \mu \mathrm{m}, 550 \mu \mathrm{m}, 580 \mu \mathrm{m}$, and $610 \mu \mathrm{m}$ are clarified in Table 2.

Table 2 Input parameters for grating structure in FullWAVE (Synopsys Inc.).

\begin{tabular}{cccc}
\hline Input CW $(\mathrm{nm})$ & Red region $(\mu \mathrm{m})$ & Blue region $(\mu \mathrm{m})$ & Structure length $(\mu \mathrm{m})$ \\
\hline 1467 & 4.2 & 1.0 & 19.8 \\
1527 & 4.5 & 1.0 & 21.0 \\
1582 & 4.8 & 1.0 & 22.2 \\
1639 & 5.1 & 1.0 & 23.4 \\
\hline
\end{tabular}




\section{Results and discussion}

\subsection{Resonant wavelengths from the perturbation of 3D printed LPFG device}

The results of resonant wavelengths from an OSA corresponding to the perturbation of 3D printed LPFG device with grating periods of $520 \mu \mathrm{m}$, $550 \mu \mathrm{m}, 580 \mu \mathrm{m}$, and $610 \mu \mathrm{m}$ LPFG device are illustrated in Fig. 4. From Fig. 4, the trend of resonant wavelength shifts with several grating periods varied from $520 \mu \mathrm{m}$ to $610 \mu \mathrm{m}$ with the increment of $10 \mu \mathrm{m}$ is observed as plotted in Fig. 5 . According to the range of the light source, there are three resonant wavelengths in each perturbation. Each of resonant wavelengths refers to different coupling modes. The experimental results show the linear relationship between the resonant wavelength and the increment of grating period, whereas the

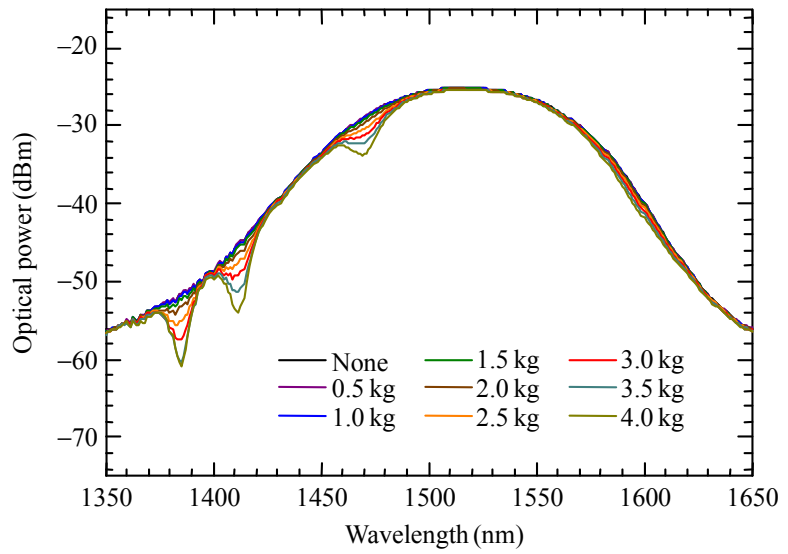

(a)

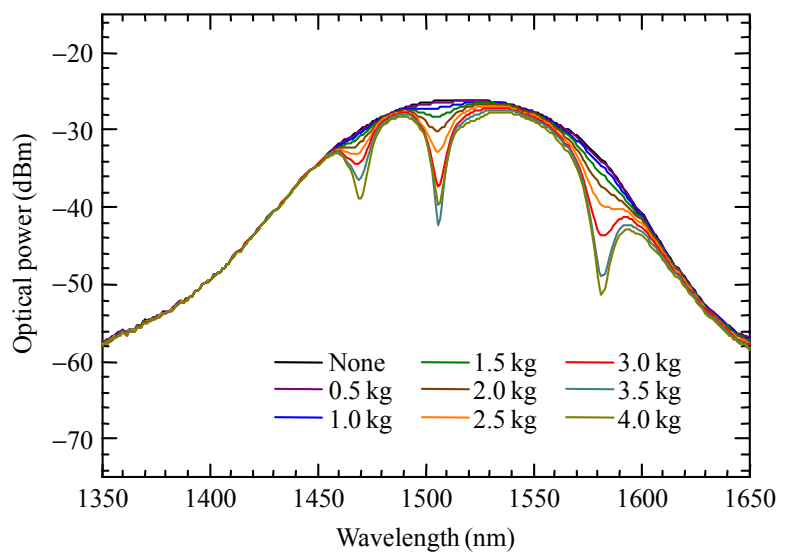

(c) higher coupled mode yields the longer shift. Results in Fig. 5 show the linear relationship between the resonant wavelength and grating period with slopes and coefficient of determination $\left(R^{2}\right)$ as $1.3345 \times 10^{-3} / 0.9984, \quad 1.5339 \times 10^{-3} / 0.9994, \quad$ and $1.8885 \times 10^{-3} / 0.9995$, respectively. Therefore, we can conclude that the third resonant wavelength yields the highest sensitivity, in other words, the higher number of coupled mode is, the higher sensitivity is [8]. The magnitude of resonant dip refers to the weight of perturbation.

The power attenuation of the resonant wavelengths shown in Fig. 4 is clarified in Fig. 6. The attenuation of all resonant wavelengths depends on grating pitches along the 3D printed LPFG device. The grating pitches are not perfectly the same as each other resulting in the non-uniform of the attenuation. However, there is a limitation of the

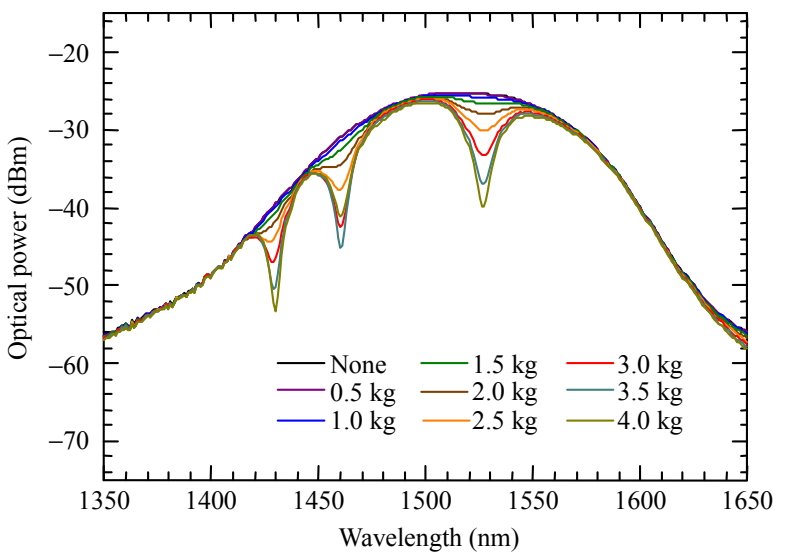

(b)

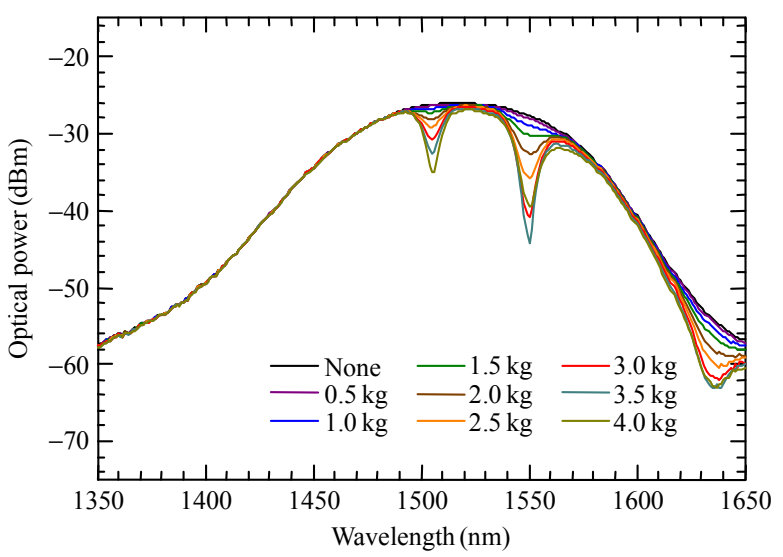

(d)

Fig. 4 Resonant wavelengths by the perturbation of 3D printed LPFG device with (a) $520 \mu \mathrm{m}$, (b) $550 \mu \mathrm{m}$, (c) $580 \mu \mathrm{m}$, and (d) $610 \mu \mathrm{m}$ grating period. 


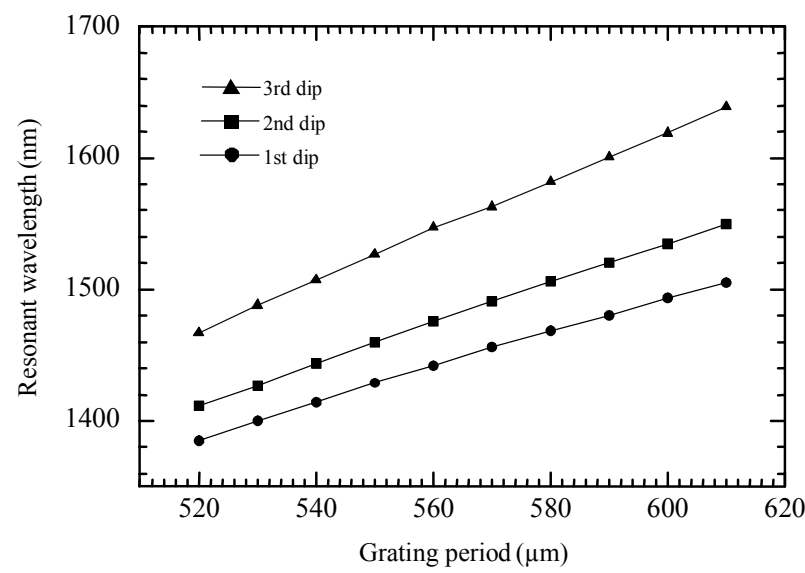

Fig. 5 Relationship between resonant wavelengths and grating periods.

perturbation weight due to vertexes of the 3D printed LPFG device as they cause the total light scattering inside the fiber when higher perturbation is applied. The third resonant wavelength in Fig. 4(a) has the shortest dip compared with others because the strength of coupling mode is not so much high at that wavelength when comparing with the first and second resonant wavelengths. Moreover, the precise dimension of grating vertexes along the device is low comparing with longer grating period or larger vertexes of the device. In case of the application in the environment with different temperatures, the grating period slightly expands when the temperature increases resulting in the resonant shift. Moreover, the resonant attenuation decreases at higher temperature due to the expansion of grating pitches resulting in the lower sensitivity. The overall resonant shift varies between $0.22 \mathrm{~nm} /{ }^{\circ} \mathrm{C}$ and $0.34 \mathrm{~nm} /{ }^{\circ} \mathrm{C}$ while its linearity shift by its coefficient of determination $\left(R^{2}\right)$ is average at 0.9883 .

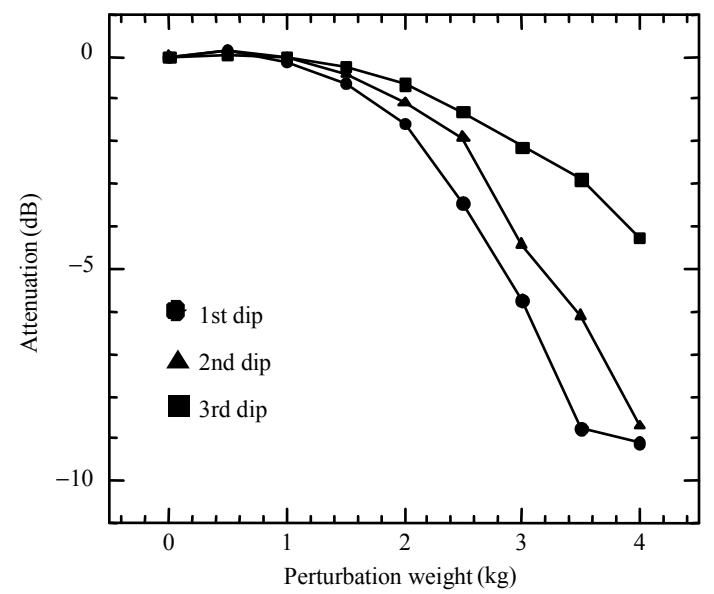

(a)

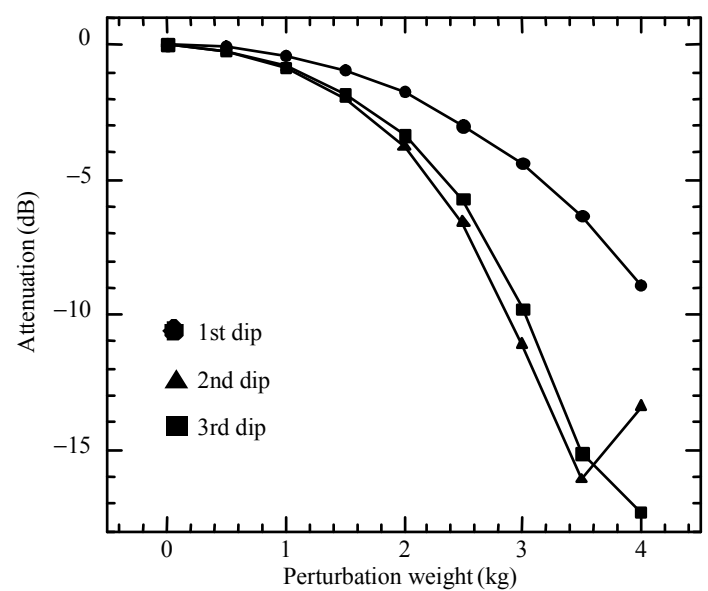

(c)

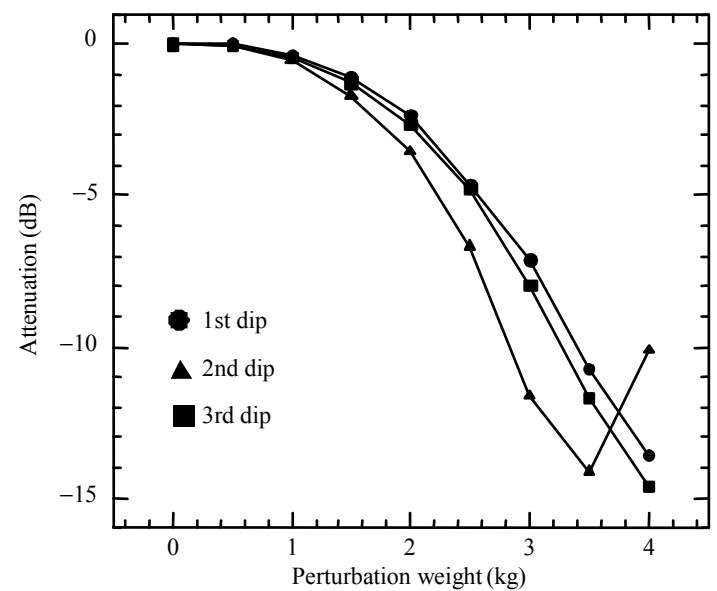

(b)

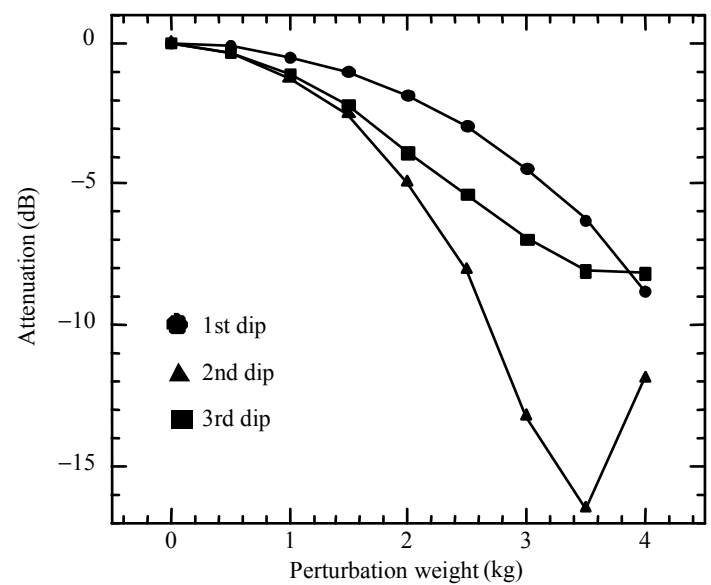

(d)

Fig. 6 Relationship between perturbation weights and the attenuation of resonant wavelengths by (a) $520 \mu \mathrm{m}$, (b) $550 \mu \mathrm{m}$, (c) $580 \mu \mathrm{m}$, and (d) $610 \mu \mathrm{m}$ grating period. 


\subsection{Simulation results of electric field distribution along grating structure}

The electric fields, compiled by FullWAVE (Synopsys Inc.) software, referring to the results of resonant wavelengths shown in Fig. 4, are illustrated in Fig. 7. The third resonant wavelength, as highest sensitivity, is chosen to be an input $\mathrm{CW}$ as listed in Table 2 for all of four structures. The electric field starts to couple to the cladding layer from the first grating pitch. The electric field distribution inside the cladding layer plays a vital role in the power attenuation inside the core layer from the corresponding wavelength. The coupling of the electric field begins when the light transmits through the first grating pitch and the optical power inside the core layer decreases simultaneously. The contour of fiber's cross section at the position of three grating pitches is illustrated in Fig. 8. The mode field has leaked into the cladding layer after passing the grating pitches. In addition, the first and the third grating pitches are measured at the central position or at $0.5 \mu \mathrm{m}$ of the pitch size, while the second grating pitch is measured at the beginning edge of its pitch region. It is clearly seen that the electric field is

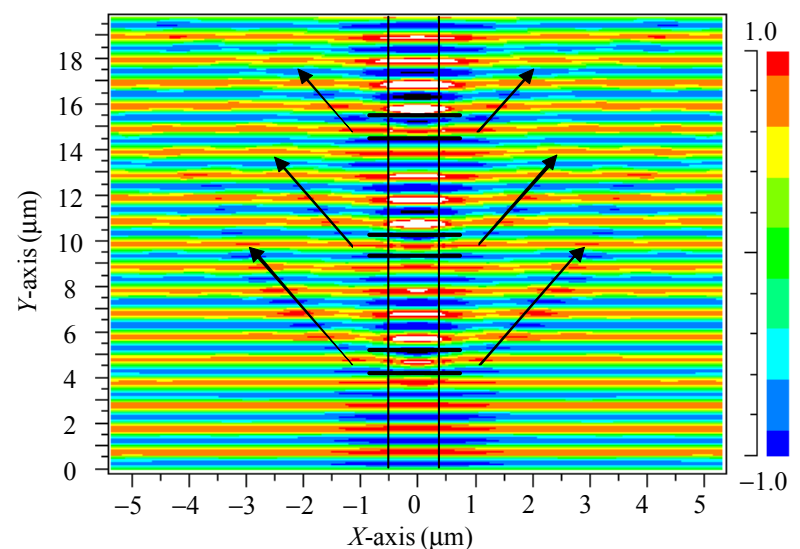

(a)

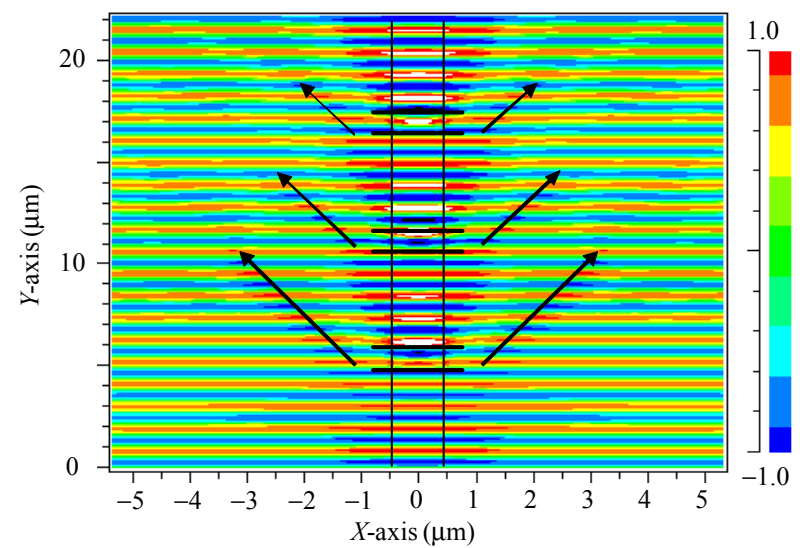

(c)

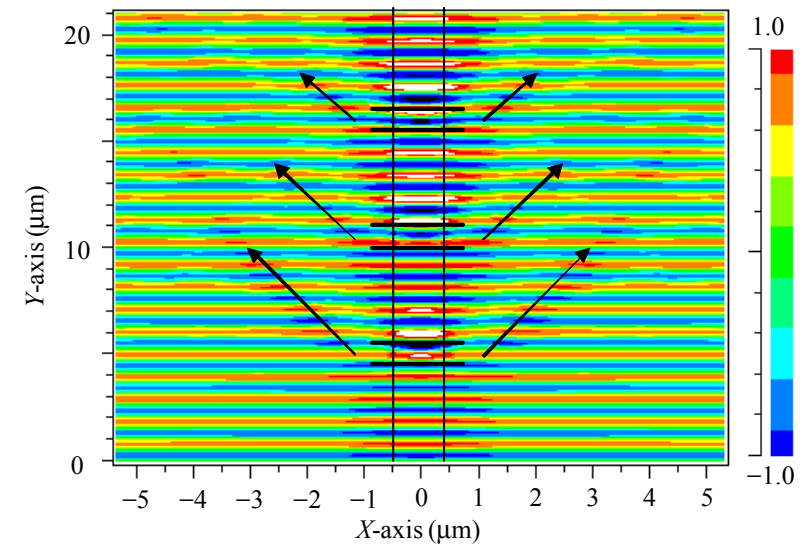

(b)

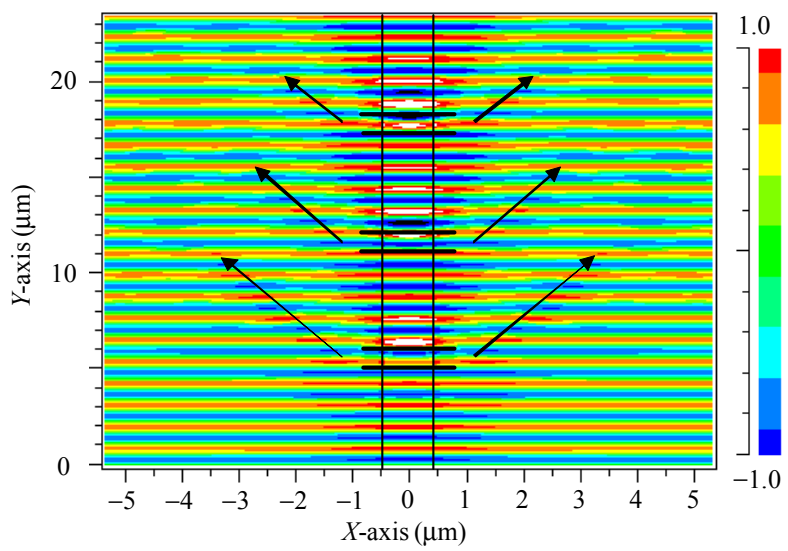

(d)

Fig. 7 Electric field distribution through the grating structure with the period of (a) $5.2 \mu \mathrm{m}$, (b) $5.5 \mu \mathrm{m}$, (c) $5.8 \mu \mathrm{m}$, and (d) $6.1 \mu \mathrm{m}$.

everywhere on the pitch and its amplitude is a function of position.

Anyway if the phase of an electric field is not in phase with the corresponding wavelength, the cladding mode might not occur as depicted in Fig. 8, as the electric field at each grating pitch still remains inside the region of core layer. The structures with the grating period of $5.2 \mu \mathrm{m}$ and $5.5 \mu \mathrm{m}$ show the clearest coupling mode at the second grating pitch in which the electric field density remains in the 
cladding region. The structure with the grating periods of $5.8 \mu \mathrm{m}$ and $6.1 \mu \mathrm{m}$ shows the clearest coupling at the first grating pitch where the electric field density remains at the core-surrounding region inside cladding layer, but the one with grating period of $5.8 \mu \mathrm{m}$ has higher and wider density regions of electric field than others. Considering the third grating pitch of the structure with $5.5 \mu \mathrm{m}, 5.8 \mu \mathrm{m}$, and $6.1 \mu \mathrm{m}$, high density of an electric field remains inside the core layer due to the very short grating length or very few amount of grating pitches inside the structure.
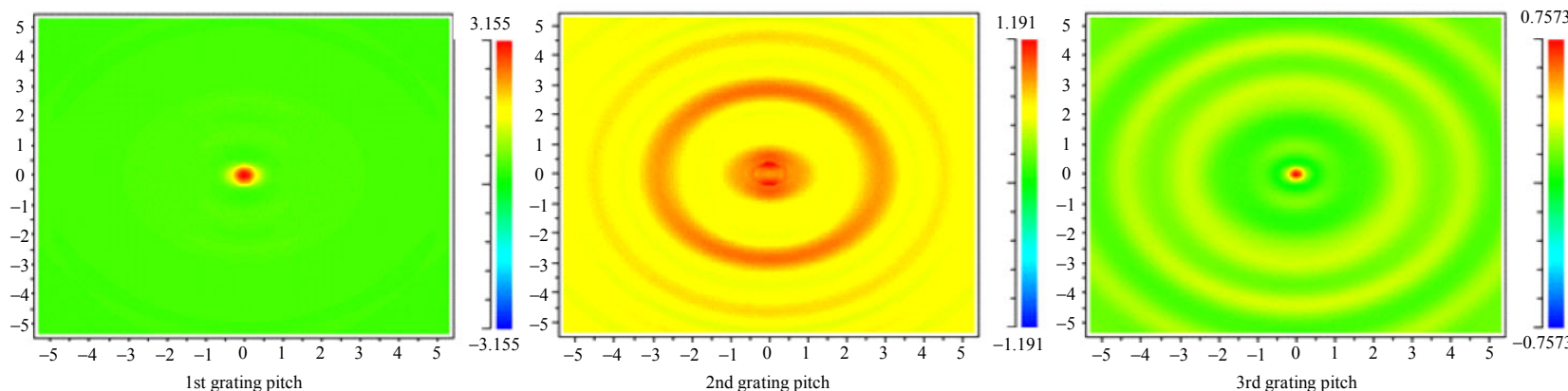

(a)
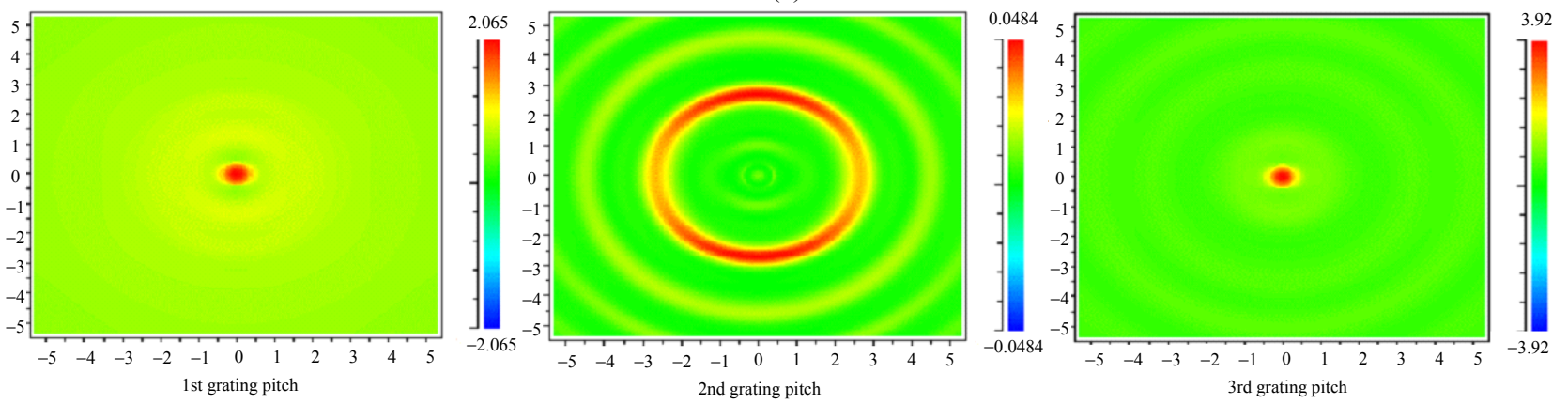

(b)
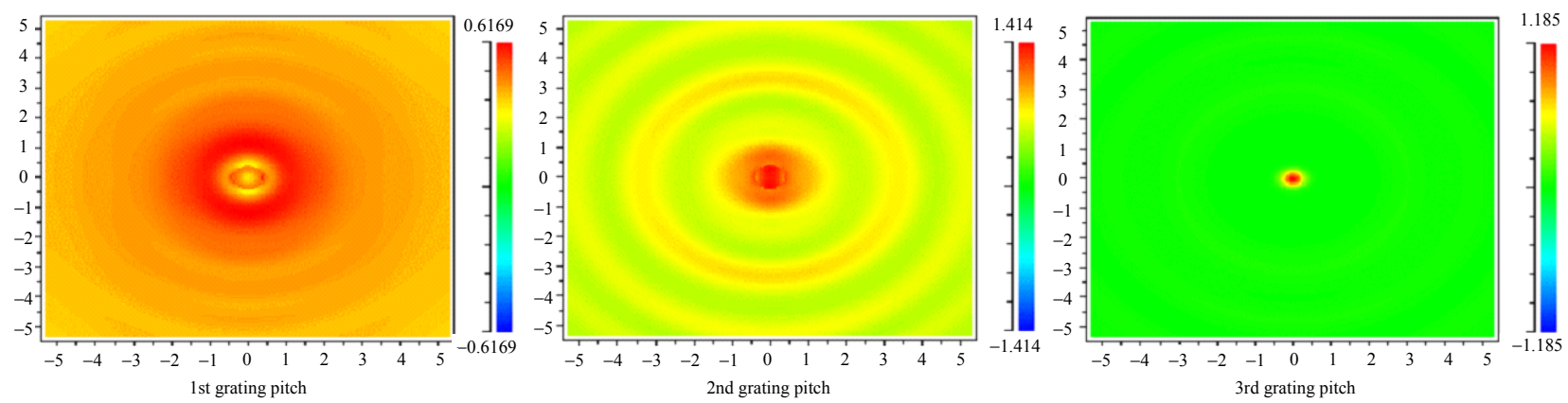

(c)
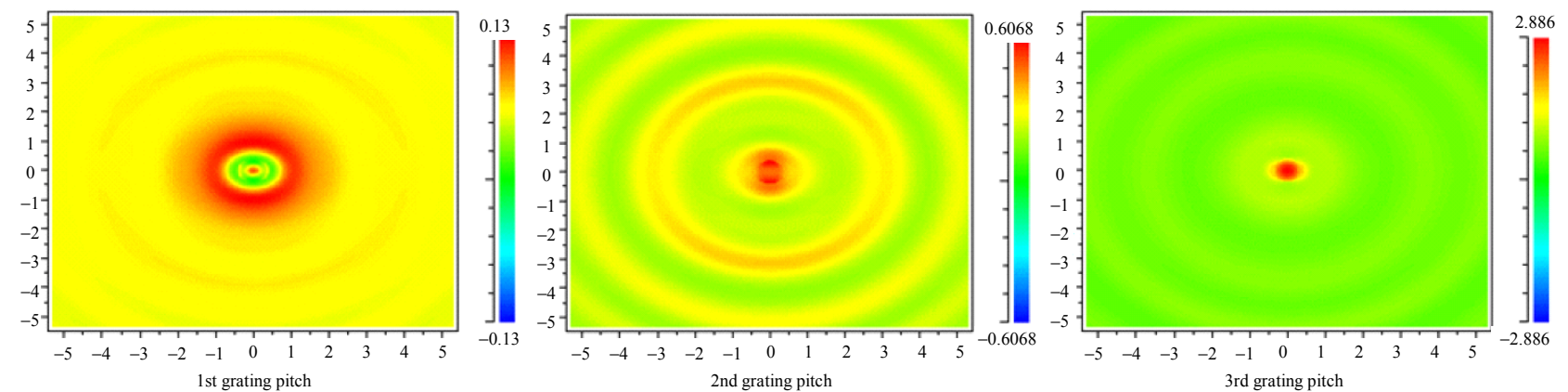

(d)

Fig. 8 Field profile of fiber's cross section at the position of the first to the third grating pitches of the structure with grating period of (a) $5.2 \mu \mathrm{m}$, (b) $5.5 \mu \mathrm{m}$, (c) $5.8 \mu \mathrm{m}$, and (d) $6.1 \mu \mathrm{m}$. 


\subsection{Discussion}

The 3D printed LPFG device has been directly applied onto the bare fiber for several times to ensure that there are no any damages or distortions on the grating vertexes. The dimension of the device is compact to protect the bending caused by the perturbation force from outside environment. The grating period below $500 \mu \mathrm{m}$ gives more curve vertexes structure, the wide bandwidth of resonant wavelength occurs and total light scattering from microbending of an optical fiber happens when few weight of perturbation is applied. The longer grating period yields more accuracy on the distance between each grating pitch. The experimental results show that the resonant shift is linear function of the size of grating period of the 3D printed LPFG device. At different temperatures, the tolerance of the spectrum at a sensor node can be set to receive the resonant shift from its original position.

The electric field distribution along fiber core passing through grating structure begins to partially scatter to the cladding layer at the first grating pitch while the other still passes throughout the core layer and scatters at the next grating pitches if that wavelength matches the coupled mode of the fiber as expressed in (2). When the attenuation reaches a point that its electric field mode is in phase with the coupling coefficient, the grating length no longer has any effect on the attenuation. The major issue to the formation of the electric field profile is the refractive indices of both core and cladding layers as they have an effect on different coupling modes in different optical fibers under the same grating period. In addition, the surrounding environment parameters, such as temperature, stress, and strain all play a vital role to the coupling coefficient of the fiber.

\section{Conclusions}

The resonant wavelengths caused by the 3D printed LPFG device are observed. From its high sensitivity, it has a great potential to be employed in selective resonant wavelengths, in other words, selective wavelength filters. In addition, characteristics of electric field distribution are simulated to observe the field interference within the cladding layer to determine the optical power loss of specific wavelengths. This proposed 3D printed LPFG device is compatible with the selective tunable filter and also a decent candidate to be used as an intruder sensor for big objects like humans or animals.

Open Access This article is distributed under the terms of the Creative Commons Attribution 4.0 International License (http://creativecommons.org/licenses/by/4.0/), which permits unrestricted use, distribution, and reproduction in any medium, provided you give appropriate credit to the original author(s) and the source, provide a link to the Creative Commons license, and indicate if changes were made.

\section{References}

[1] P. Sehgal and H. Dua, "A review on optical fiber long period grating, its application in optical communication system," International Journal of Advanced Research in Computer and Communication Engineering, 2015, 4(5): 624-630.

[2] A. M. Vengsarkar, P. J. Lernaire, J. B. Judkins, V. Bhatia, T. Erdogan, and J. E. Sipe, "Long-period fiber gratings as band-rejection filters," Journal of Lightwave Technology, 1996, 14(1): 58-65.

[3] D. D. Davis, T. K. Gaylord, E. N. Glytsis, S. G. Kosinski, S. C. Mettler, and A. M. Vengsarkar, "Long-period fibre grating fabrication with focused $\mathrm{CO}_{2}$ laser pulses," Electronics Letters, 1998, 34(3): 302-303.

[4] S. Savin, M. J. F. Digonnet, G. S. Kino, and H. J. Shaw, "Tunable mechanically induced long-period fiber gratings," Optics Letters, 2000, 25(10): $710-712$.

[5] R. Khun-In, M. Takagi, K. Nanjo, Y. Jiraraksopakun, A. Bhatranand, and H. Yokoi, "Resonant wavelength observation by $3 \mathrm{D}$ printed mechanically induced long-period fiber grating device," Advanced Photonics Congress, Switzerland, July 2-5, 2018.

[6] T. Yokouchi, Y. Suzaki, K. Nakagawa, M. Yamauchi, M. Kimura, Y. Mizutani, et al., "Thermal tuning of mechanically induced long-period fiber grating," Applied Optics, 2005, 44(24): 5024-5028.

[7] Z. Gu, Y. Shi, and K. Gao, "Dispersion characteristics in metal coated long period gratings," Optical and Quantum Electronics, 2012, 44(3-5): 303-311.

[8] S. W. James and R. P. Tatam, "Optical fibre long-period grating sensors: characteristics and application," Measurement Science and Technology, 2003, 14(5): 49-61.

[9] Synopsys Inc., "RSoft FuLLWAVE v2018.03 User Guide," New York: Optical Solutions Group, 2018: 9-13.

[10] H. L. Tseng, E. Chen, H. Rong, and N. Na, "High-performance silicon-on-insulator grating coupler with completely vertical emission," Optical Express, 2015, 23(19): 24433-24439. 